\title{
Comparative study for osseointegration according to surface treatments of dental implants
}

\author{
Myung Hyun Jun ${ }^{1}$, Myoung-Ju Kim² ${ }^{2}$ You-Song $\mathrm{Sim}^{2}$, and Min-Suk Kook ${ }^{3 *}$ \\ ${ }^{1}$ Master's Student, Department of Oral and Maxillofacial Surgery, College of Dentistry, Chonnam National University, Gwangju, Republic of Korea \\ ${ }^{2}$ Ph.D. Student, Department of Oral and Maxillofacial Surgery, College of Dentistry, Chonnam National University, Gwangju, Republic of Korea \\ ${ }^{3}$ Professor, Department of Oral and Maxillofacial Surgery, College of Dentistry, Chonnam National University, Gwangju, Republic of Korea
}

\begin{abstract}
The purpose of this study was to evaluate the effect of hydroxyapatite (HA) full-coating and resorbable blast media (RBM) on the osseointegration of implants placed in the bone. Twelve rabbits were used as experimental animals and three implants were installed in each tibia. Rabbits in the control group were provided with no additional treatments to the fixture. Rabbits in experimental group I were provided with RBM treatment and rabbits in experimental group II were provided with full HA coating. The animals were sacrificed 4 weeks after implantation. The parts of the tibia containing the implant were harvested and radiographs were acquired. Specimens were prepared for histological examination and histomorphometric analysis of the bone-to-implant contact (BIC) ratio. The extent of osseointegration of the implants was different among the groups. Histomorphometrically, the full HA coating group showed the highest BIC ratio, followed by the RBM treatment and the control group. The three groups significantly differed when using the Kruskal-Wallis test and Mann-Whitney U-test. These results suggest that the full HA coating and RBM treatment to the fixture can provide significantly higher osseointegration. The full HA coating provided more osseointegration than the RBM treatment.
\end{abstract}

Key Words: Dental implants, Durapitite, Osseointegration, Surface properties

(c) This is an open-access article distributed under the terms of the Creative Commons Attribution Non-Commercial License (http://creativecommons.org/licenses/by-nc/4.0) which permits unrestricted noncommercial use, distribution, and reproduction in any medium, provided the original work is properly cited.

\section{Introduction}

Implant surgery involves replacing tooth function by planting an artificial tooth with a root-like artificial fixture to the tooth defect area, replacing the natural tooth. It has become a common dental surgery worldwide. In edentulous patients, bone type IV or lack of bone has been overcome to increase early bone contact by increasing osteogenesis and the amount of blood flow to induce adhesion in the bone graft [1]. Rapid adhesion is the main purpose of surface treatment of dental implants. Moreover, the surface of dental implants is the most important factor influencing the mechanical strength of the interface between the implant and the tissue [2].

Several trials to increase bone adhesion by surface treatment of implants have been conducted, including hydroxyapatite (HA) full-coating and resorbable blast media (RBM) treatment $[3,4]$. HA initiates early healing in implants and combines with bone. It has an ability for protein adhesion to the surface; therefore, it accelerates the healing course of

Received May 1, 2020; Revised May 30, 2020; Accepted June 5, 2020

${ }^{*}$ Corresponding author: Min-Suk Kook, Department of Oral and Maxillofacial Surgery, College of Dentistry, Chonnam National University, 33 Yongbong-ro, Buk-gu, Gwangju 61186, Republic of Korea.

Tel: +82-62-220-5439, Fax: +82-62-228-8712, E-mail: omskook@jnu.ac.kr

Copyright $\odot$ 2020, Oral Biology Research Institute 
adhesive proteins, such as fibrinogen. HA full-coating commonly uses the method of plasma-spray-coating, which was applied in a cone-shaped integral implant for the first time in 1990 in Calcitek [5]. However, limitations of HA full-coating are loosening of titanium molecules by the absorption of HA, possibility of poor adhesion, and chances of failure in infection control [6]. Moreover, the implant surface can be worn down by spraying rough particles of various diameters.

In RBM treatment, the possibility of poor bone adhesion by residual surface particles in early oxide alumina particle usage was solved by biodegradable calcium phosphate particles [7]. A study has been conducted on the bone-remedy patterns in the cerebral cortex and bone marrow compartment by implantation in rabbits, regarding this surface treatment [8].

In this study, two types of surface treatment of implants in rabbits' tibia were applied to compare the extent of bone adhesion and their patterns.

\section{Materials and Methods}

\section{Animal experiment}

This study was performed in accordance with the Institutional Guidelines for Experimental Animal Care and Use of Chonnam National University Hospital (CNUH IACUC-18022).

For the experiment, 12 male rabbits, weighing approximately $2 \mathrm{~kg}$, were grown in the same condition. We chose 36 implants, measuring $2.75 \times 10 \mathrm{~mm}$ (KJ Meditech, Gwangju, Korea), and divided them into groups of no treatment (control group), RBM surface treatment (group 1), and HA surface treatment (group 2) (Fig. 1).

All surgical tools were sterilized in an autoclave 24 hours before the experiment. After weighing the rabbits, $3 \mathrm{~mL} /$ kg of Zoletil (Zoletil50; Virbac Co, Carros, France) was infiltrated in the femoral region for sedation and anesthesia. After shaving the right tibial parts, rabbits were placed in the prone position, and the operation site was disinfected with povidone-iodine, chlorhexidine gluconate solution, and isopropanol. After infiltrative anesthesia at the operation site with lidocaine containing epinephrine, incision with a \#15 blade to peel the tissue and periosteum led to the exposure of tibia. With the exposure of tibia, bone formation for implantation with irrigation was performed with a guide or twist drill. When completing bone formation, implantation into the right tibia of 12 rabbits under droplets of saline solution was performed using an implant kit. Of all 36 implants, 12 were placed into each group of rabbits. A periosteal suture using 2-0 Vicryl after implantation was placed, followed by a 3-0 Vicryl muscle suture. Finally, 5-0 and 6-0 nylon threads were used for subcuticular and skin sutures. In the same way as before the operation, the operation site was disinfected with povidone-iodine, chlorhexidine gluconate solution, and isopropanol. Keromin,a firstgeneration cephalosporine, was injected.

\section{Biopsy and radiographic inspection}

The whole-body condition of the rabbit, scar healing, and infection control of the operation site were monitored every day before disinfection. The rabbits were sacrificed after 4 weeks with a $\mathrm{KCl}$ solution. With a margin of more than $3 \mathrm{~mm}$ around the placed implants, samples were prepared after bone fragmentation with bone knives. Radiographs of all samples were acquired with bite films to check the position of implants and bone adhesion condition of the perpendicular bone-cutting samples. Using parallel radiography at $60 \mathrm{kVp}$ and $6 \mathrm{~mA}$, a standard X-ray image was

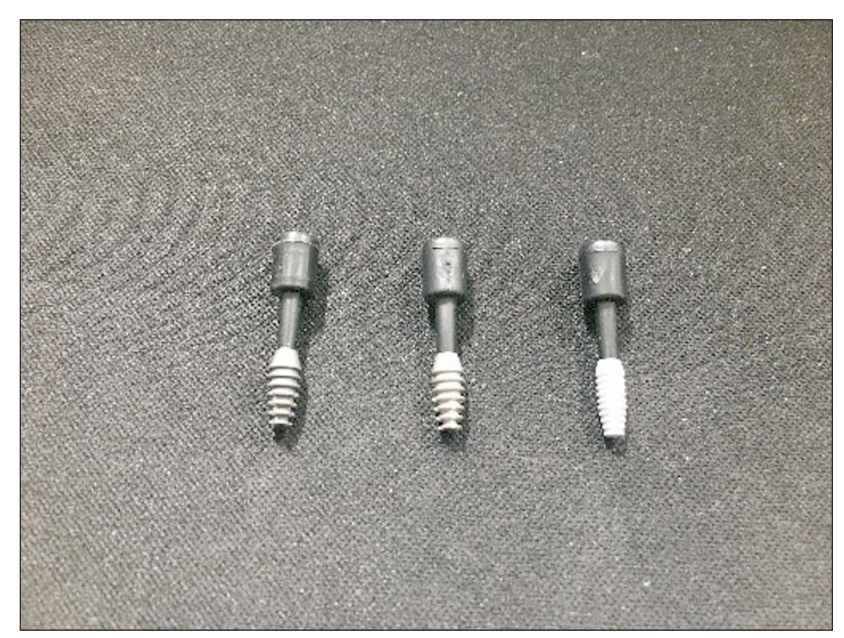

Fig. 1. Dental implants used in the experiment (left: smooth surface; middle: resorbable blast media-treated surface; right: hydroxyapatitetreated surface). 
acquired at a subject-to-lens distance of $15 \mathrm{~cm}$ after setting the fixed value. Checking the position of implants and their axis, preprocessing was performed for a histological analysis. After fixation with Schaffer's solution (2/3 of $96 \%$ ethanol $+1 / 3$ of $37 \%$ formaldehyde), no demineralization or embedding, samples were stiffened in methylmethacrylate after the dehydration process. The sample was cut twice with 300- $\mu \mathrm{m}$ thickness with the direction of the long axis at the thickest part of the implant. Subsequently, samples were grinded with a grinder-polisher (Metaserv; Buehler, Lake Bluff, IL, USA) until their thickness became $50 \mu \mathrm{m}$. For histological observation, samples were stained with the Goldner's trichrome. Using an optical microscope, the extent and appearance of bone adhesion around the implant were analyzed.

\section{Histomorphometric analysis}

A histomorphometric assessment of each sample was performed around the tibia, and an image enlarged 40 times in the thread of the screw in each sample was saved digitally. A histomorphometric analysis of the bone-to-implant contact (BIC) ratio between the implant and the bone margin was performed with the Scion Image (Scion Co., Frederick, MD, USA). The statistical test included comparing the equivalent values and post-hoc comparison using the Kruskal-Wallis test and Mann-Whitney U-test.

BIC ratio $(\%)=($ the total length of direct contact implant surface)/(the total length of implant surface)*100

\section{Results}

\section{Gross findings}

The area of the implant healed properly, with no findings of dehiscence, infection, or bone fracture in gross findings for 4 weeks. Moreover, there were no external exposures or specific inflammation, and implants healed well.

\section{Radiographic findings}

Radiopacity was found in the cortical bone in all groups while the marrow size differed with the object. The marrow showed a higher radiolucency in the control group and experimental group 2 than in experimental group 1. Most implants were found through the tibia, penetrating through the lower part of the bone. The location of implant varies with the thickness of the cortical bone and size of the bone marrow, as good bone adhesion is found.

\section{Histological findings}

A thick cortical bone was found in every sample of tibia in the control group while slight bone trabecula was found in the bone marrow. The thickness of the cortical bone differed with the object while implants mostly looked wellprogressed without intervention of soft tissues. A thin layer of bone adhesion was found in some implants exposed into the bone marrow (Fig. 2).

In the Experimental group 1, a thick cortical bone was found similar to the control group while some newly formed bone was found in the bone marrow. The thread
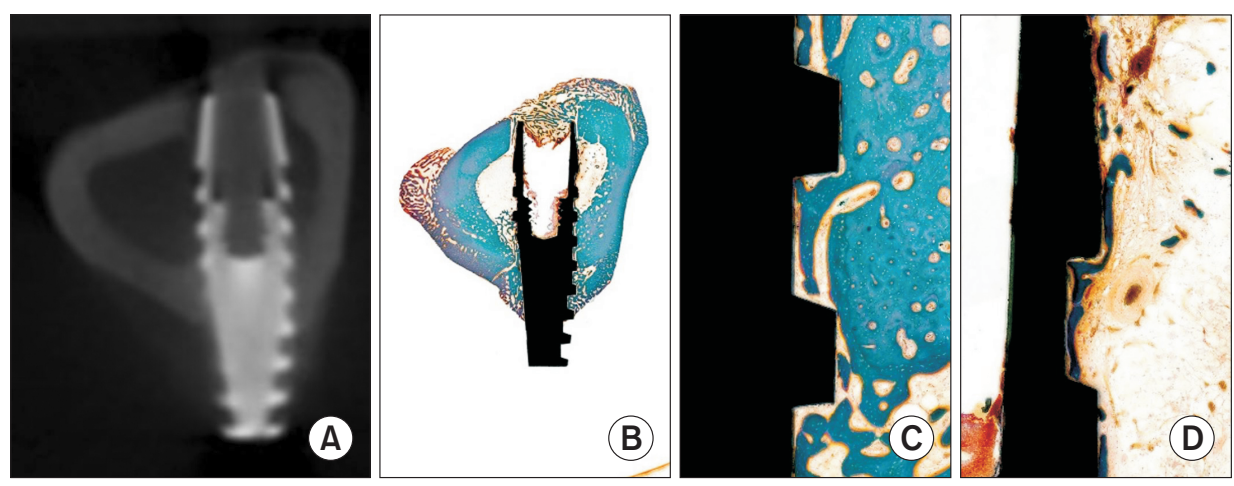

Fig. 2. The radiographic and histological photographs in the control group. (A) The tibia shows a thick cortical bone and bone marrow. (B) Implants are located in the tibia. (C) The cortical bone area shows the thread of the implant. (D) The bone marrow area shows the surface of the implant contacting the cancellous bone. 

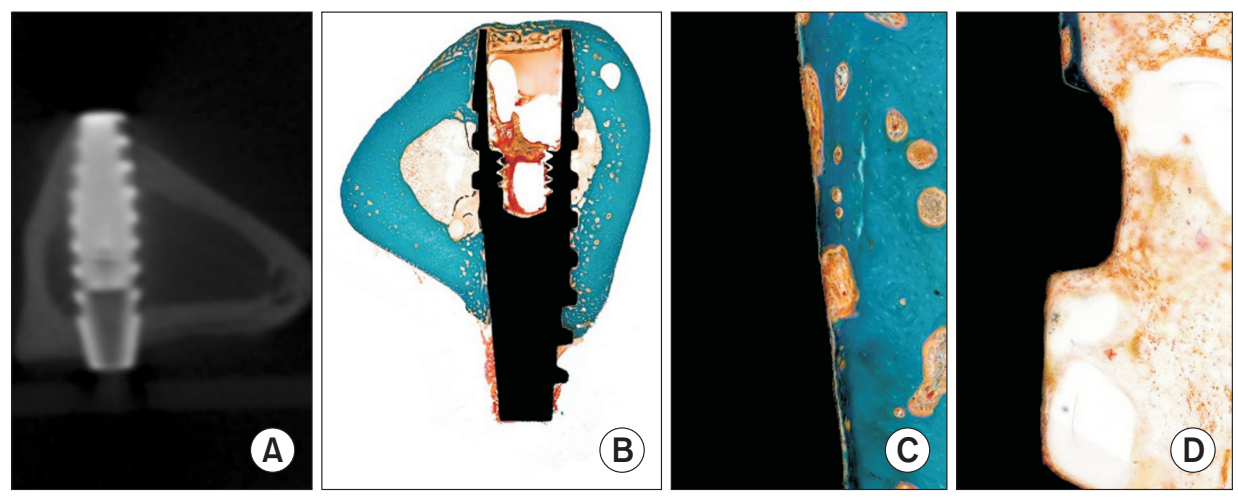

Fig. 3. The radiographic and histological photographs in experimental group 1. (A) The tibia shows a thick cortical bone and bone marrow. (B) Implants are located in the tibia. (C) The cortical bone area shows the thread of the implant filled with the cortical bone. (D) The bone marrow area shows the surface of the implant contacting the cancellous bone.
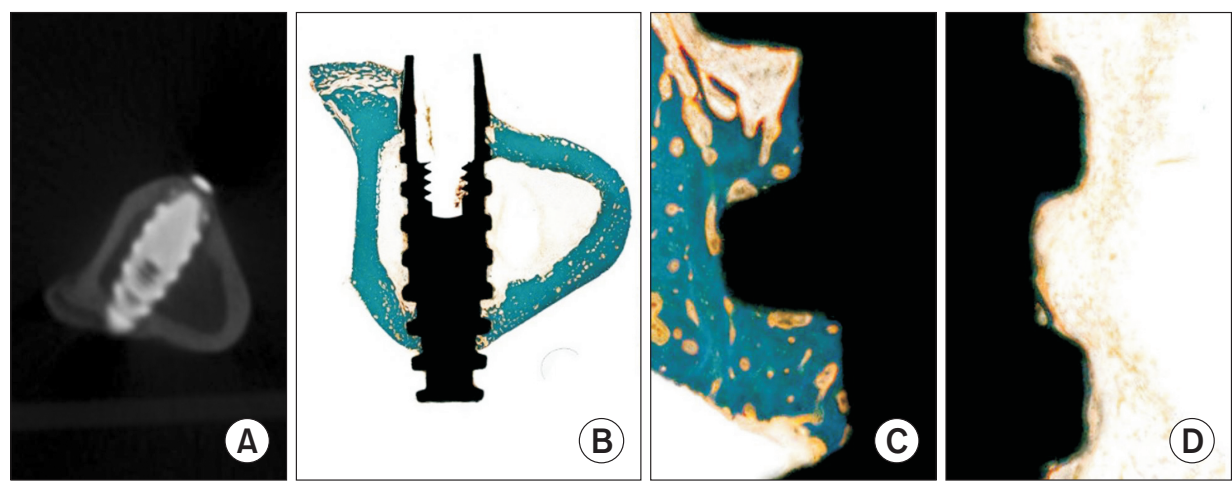

Fig. 4. The radiographic and histological photographs in experimental group 2. (A) The tibia shows a thick cortical bone and bone marrow. (B) Implants are located in the tibia. (C) The cortical bone area shows the thread of the implant filled with the cortical bone. (D) The bone marrow area shows the surface of the implant contacting the cancellous bone.

of the implant in the cortical bone area showed good bone adhesion along the implant surface. The loose bone trabecula contacted the part of the implant exposed in the bone marrow while some parts did not contact the bone (Fig. 3).

In the Experimental group 2, a thick cortical bone was found similar to the control group while some newly formed bone was found in the bone marrow. The thread of the implant in the cortical bone area showed good bone adhesion along the implant surface. The dense bone trabecula contacted the part of the implant exposed in the bone marrow while some parts did not contact the bone (Fig. 4).

\section{Histomorphometric finding}

As for the rate of the average bone contact to the surface of the implant, the control group showed the lowest rate $(32.8 \% \pm 5.16 \%)$, followed by experimental group $1(36.3 \% \pm 7.93 \%)$ and experimental group $2(45.5 \% \pm$ 8.05\%). Using IBM SPSS Statistics ver. 23.0 software (IBM Co., Armonk, NY, USA), statistically significant differences

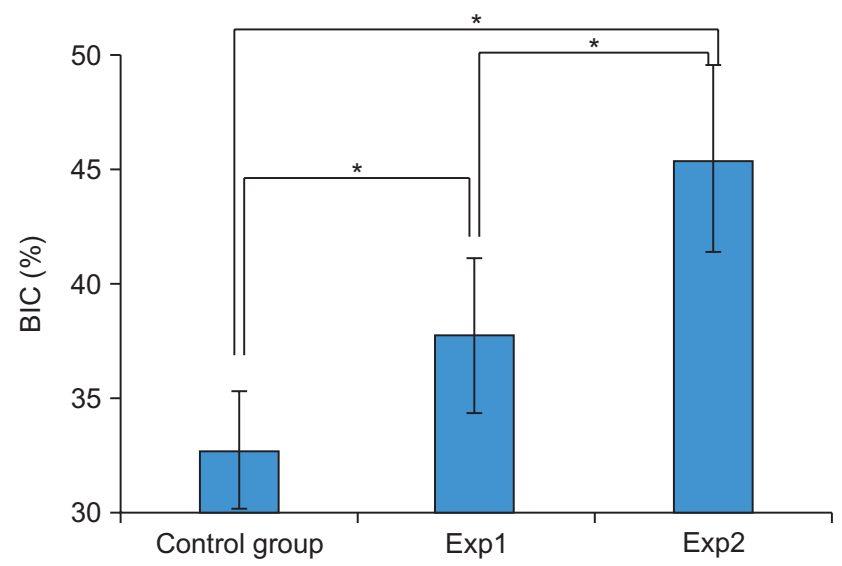

Fig. 5. This shows the extent of bone-to-implant contact (BIC) in each group. Hydroxyapatite surface treatment (experimental group 2 ) shows the highest bone contact while the control group shows the lowest $\left({ }^{*} p<0.05\right)$.

between groups were found in the Kruskal-Wallis test $(p<0.05)$.

The post-analysis performed between every two groups using the Mann-Whitney U-test showed statistically significant differences among all groups (Fig. 5). 


\section{Discussion}

Early stability is indispensable for bone adhesion after implant surgery. Factors affecting the stability include surgical technique, bone formation, extent of bone quality, surface treatment, etc [9]. According to Lekholm, the bone quality in implants is classified into four stages from I to IV clinically [10]. Whether or not implant surgery is successfully performed largely depends on the bone quality of the mandible, as the mandible has a denser cortical bone than the maxilla. Both the mandible and maxilla show decreased thickness of the cortical bone and increased porosity of the bone trabecula on the rear side, which affects the early success rate of the implant. Class IV bone has low density and solidity and a thinner layer of cortical bone surrounding the cancellous bone, which is mainly found on the rear side of the maxilla and mandible of patients with osteoporosis. Previous studies showed increased implant stability with increased thickness of the cortical bone but decreased thickness of the cancellous bone in implant surgery [11]. Similarly, our study showed better bone adhesion in the cortical bone than in the cancellous bone on radiographic and histological inspections.

Many clinical trials and research to increase bone adhesion through various surface treatments have been evaluated clinically. It is well-known that HA coating provides biocompatibility to the fixture, which decreases the time for osseointegration [12]. HA also increases the adhesion strength between serum proteins and growth factors, which accelerates adhesion and proliferation of bone cells. Finally, it reduces the time for implant loading in cases of poor bone quality, such as class IV bone. Moreover, in this study, the HA coating treatment showed the highest BIC ratio.

RBM treatment has been used to promote osseointegration by roughening the surface of the fixture by spraying biodegradable calcium phosphate [13]. It is well-known that RBM treatment blocks early bone resorption and retains the stability of the fixture after implant placement, which shows low bone resorption and old lamellar bone [14]. In this study, the higher BIC rate with RBM treatment than the control verifies this tendency.

Particularly, HA coating showed more significant results compared to RBM surface treatment. RBM surface treatment provides marked hydrophobicity while HA coating decreases the contact angle [4]. Thus, fast hydration of the oxide layer by hydrophilicity may promote contact of the biomolecules and improve arrangement of the bone tissue. This HA coating layer of enhanced wetting increases osseointegration. Therefore, for class IV bone or poor bone quality, such as in osteoporosis, implants with HA coating increase early bone contact in implant surgery and enable implant stability. Various trials of HA coating and other studies related to it should be conducted.

In a study on HA coating, the solvent casting process of nano-sized particles, such as Poly (lactide-co-glycolide)/ bioactive glass/HA, was used. In this case, the ideal surface for adhesion and survival of stem cells was offered, and antimicrobial action was triggered [15]. Aseptic loosening, the main cause of failure of titanium-based implants, was resolved by increasing the biologic stability by fixation of the covalent bone at each level attained by stable collagen/HA multi-layered modified titanium coating [16]. According to the study by Oliver et al. [18], after forming an amorphous layer of calcium phosphate in bioactive glass, crystallization of the amorphous matrix by osteoblasts produced a new hydroxyl carbonated apatite layer. With this coating substance, the glass-ceramic complex coating method, which adds a ceramic substance to the bioactive glass, or glasspolymer complex coating method, which uses polymers, such as keto acid or polycaprolactone, has been used in research. Regardless of these methods, the original enameling method is replaced by the plasma-spraying method, or electrophoretic deposition is used as an alternative in other studies on the coating method [17].

With the average BIC ratio, the HA surface treatment shows the best results. Therefore, we may expect a greater success rate of implants with HA surface treatment clinically.

\section{Acknowledgements}

The authors do not have any commercial associations, either currently or within the past 5 years, that might pose any potential, perceived, or real conflicts of interest for conducting or publicizing the study. 


\section{Conflicts of Interest}

The authors declare that they have no competing interests.

\section{ORCID}

\author{
Myung-Hyun Jun \\ https://orcid.org/0000-0002-2944-7573 \\ Myoung-Ju Kim \\ https://orcid.org/0000-0003-4082-2765 \\ You-Song Sim \\ https://orcid.org/0000-0003-1603-3248 \\ Min-Suk Kook \\ https://orcid.org/0000-0002-8053-8534
}

\section{References}

1. Hutton JE, Heath MR, Chai JY, Harnett J, Jemt T, Johns RB, McKenna S, McNamara DC, van Steenberghe D, Taylor R, Watson RM, Herrmann I. Factors related to success and failure rates at 3-year follow-up in a multicenter study of overdentures supported by Brånemark implants. Int J Oral Maxillofac Implants 1995;10:33-42.

2. Eriksson C, Lausmaa J, Nygren H. Interactions between human whole blood and modified TiO2-surfaces: influence of surface topography and oxide thickness on leukocyte adhesion and activation. Biomaterials 2001;22:1987-1996. doi: 10.1016/s0142-9612(00)00382-3.

3. Yaman F, Ağaçayak S, Atilgan S, Benlidayi E, Ucan MC, Erol B, Kaya B, Gunay A, Guven S. Effects of systemic zoledronic Acid administration on osseointegration of hydroxyapatite-coated and resorbable blast material surface implants in rabbit models. Int J Oral Maxillofac Implants 2012;27:1443-1447.

4. Jung UW, Hwang JW, Choi DY, Hu KS, Kwon MK, Choi SH, Kim HJ. Surface characteristics of a novel hydroxyapatite-coated dental implant. J Periodontal Implant Sci 2012;42:59-63. doi: 10.5051/jpis.2012.42.2.59.

5. Watson CJ, Tinsley D, Ogden AR, Russell JL, Mulay S, Davison EM. A 3 to 4 year study of single tooth hydroxylapatite coated endosseous dental implants. Br Dent J 1999;187:9094. doi: 10.1038/sj.bdj.4800211.

6. Abraham CM. A brief historical perspective on dental implants, their surface coatings and treatments. Open Dent J 2014;8:50-55. doi: 10.2174/1874210601408010050.
7. Lee YJ, Lee BU, Kim YS. Current studies of implant surface treatment- in perspective of bone healing mechanism. Implantology 2003;12:12-29.

8. Soto-Peñaloza D, Caneva M, Viña-Almunia J, Martín-deLlano JJ, Peñarrocha-Oltra D, Peñarrocha-Diago M. Bonehealing pattern on the surface of titanium implants at cortical and marrow compartments in two topographic sites: an experimental study in rabbits. Materials (Basel) 2018;12:85. doi: 10.3390/ma12010085.

9. Pai UY, Rodrigues SJ, Talreja KS, Mundathaje M. Osseodensification- a novel approach in implant dentistry. J Indian Prosthodont Soc 2018;18:196-200. doi: 10.4103/jips. jips_292_17.

10. Lekholm U, Zarb GA. Patient selection and preparation. In: Branemark PI, Zarb GA, Albrektsson T, editors. Tissueintegrated prostheses: osseointegration in clinical dentistry. Chicago: Quintessence; 1985. p. 199-209.

11. Jaffin RA, Berman CL. The excessive loss of Branemark fixtures in type IV bone: a 5-year analysis. J Periodontol 1991;62:2-4. doi: 10.1902/jop.1991.62.1.2.

12. Jeong KI, Kim YK, Moon SW, Kim SG, Lim SC, Yun PY. Histologic analysis of resorbable blasting media surface implants retrieved from humans: a report of two cases. J Korean Assoc Oral Maxillofac Surg 2016;42:38-42. doi: 10.5125/jkaoms.2016.42.1.38.

13. Vercaigne S, Wolke JG, Naert I, Jansen JA. A histological evaluation of $\mathrm{TiO} 2$-gritblasted and $\mathrm{Ca}-\mathrm{P}$ magnetron sputter coated implants placed into the trabecular bone of the goat: part 2. Clin Oral Implants Res 2000;11:314-324. doi: 10.1034/j.1600-0501.2000.011004314.x.

14. Gansukh O, Jeong JW, Kim JW, Lee JH, Kim TW. Mechanical and histological effects of resorbable blasting media surface treatment on the initial stability of orthodontic mini-implants. Biomed Res Int 2016;2016:7520959. doi: 10.1155/2016/7520959.

15. Mehdikhani-Nahrkhalaji M, Fathi MH, Mortazavi V, Mousavi SB, Hashemi-Beni B, Razavi SM. Novel nanocomposite coating for dental implant applications in vitro and in vivo evaluation. J Mater Sci Mater Med 2012;23:485-495. doi: 10.1007/s10856-011-4507-0.

16. Ao H, Zong J, Nie Y, Wan Y, Zheng X. An in vivo study on the effect of coating stability on osteointegration performance of collagen/hyaluronic acid multilayer modified titanium implants. Bioact Mater 2017;3:97-101. doi: 10.1016/j.bioactmat.2017.07.004.

17. Oliver JN, Su Y, Lu X, Kuo PH, Du J, Zhu D. Bioactive glass coatings on metallic implants for biomedical applications. Bioact Mater 2019;4:261-270. doi: 10.1016/ j.bioactmat.2019.09.002. 